\title{
Tuberculosis espinal lumbosacra y craneocervical: Un clásico imitador en dos localizaciones atípicas
}

\author{
Dres. Amadeo Muntané $S^{(1)}$, Albert Pons $E^{(1)}$, Joana Valcarcel $J^{(1)}$, Lucía Aja $R^{(1)}$, Xavier Sanjuan $G^{(2)}$.
}

1. Servicio de Radiodiagnóstico, Departamento de Neurorradiología, Hospital Universitari de Bellvitge. L'Hospitalet de Llobregat, Barcelona - España.

2. Servicio de Anatomía Patológica, Departamento de Patología de hueso y partes blandas, Hospital Universitari de Bellvitge. L'Hospitalet de Llobregat, Barcelona - España.

Isolated spinal tuberculosis in the lumbosacral and cranio-cervical hinge regions: a classic copycat in two atypical regions.

\begin{abstract}
Two cases of spinal tuberculosis in atypical regions are presented; in the lumbosacral hinge and cranio-cervical regions respectively; focusing on the radiological appearance using Computed Tomography (CT) and Magnetic Resonance. With regard to these atypical presentations, the clinical history, epidemiology and radiology of spinal tuberculosis are reviewed.
\end{abstract}

Keywords: CT scan, MRI, spine, Tuberculosis.

Resumen: Se presentan dos casos de tuberculosis espinal en localizaciones atípicas; la charnela lumbosacra y cráneo-cervical respectivamente; centrándonos en su aspecto radiológico por tomografía computarizada (TC) y resonancia magnética. A propósito de estas atípicas presentaciones se revisa la clínica, epidemiología y radiología de la tuberculosis en la columna vertebral.

Palabras clave: Columna vertebral, Resonancia magnética, Tomografía computarizada, Tuberculosis.

Muntané A, Pons A, Valcarcel J, Aja L, Sanjuan X. Tuberculosis espinal lumbosacra y cráneo cervical: Un clásico imitador en dos localizaciones atípicas. Rev Chil Radiol 2015; 21(2): 79-82.

Correspondencia: Dr. Albert Pons E. / aponse@hotmail.com

Trabajo recibido el 27 de agosto de 2014. Aceptado para publicación el 01 de diciembre de 2014.

\section{Introducción}

Se expone el caso de dos pacientes sin antecedentes de interés que consultaron por dolor lumbar y cervical, respectivamente, de meses de evolución. Las pruebas de imagen y los posteriores estudios patológicos confirmaron el diagnóstico de tuberculosis lumbo-sacra y de la unión craneo-cervical. A propósito de estas atípicas localizaciones; se revisa la clínica, epidemiología y radiología de la tuberculosis en la columna vertebral, y la literatura previa sobre estas dos raras presentaciones.

\section{Presentación de los casos}

Caso 1

Mujer de 31 años, de origen marroquí, sin antecedentes de interés. Acudió por dolor creciente en región lumbar baja con irradiación a extremidades inferiores.

Se realizó una TC que mostró una extensa destrucción ósea con gran componente de partes blandas centrado alrededor de S1 y L5 y que condicionaba estenosis de canal raquídeo. Una RM posterior delimitó mejor el componente de partes blandas, cuyo seno mostraba muy alta señal en secuencias T2 sugiriendo extensas áreas de necrosis o liqüefacción (Figura 1).

Los diagnósticos considerados fueron el cordoma y un proceso inflamatorio-infeccioso atípico de carácter granulomatoso. La opción de metástasis parecía menos probable por la edad de la paciente.

Se practicó una biopsia guiada por tomografía computarizada (TC). En las muestras obtenidas se observó una inflamación crónica granulomatosa de tipo tuberculoide (Figura 2).

Posteriormente, en las pruebas microbiológicas se aisló Mycobacterium Tuberculosis.

Se inició tratamiento médico con buena respuesta clínica y radiológica en controles posteriores. 

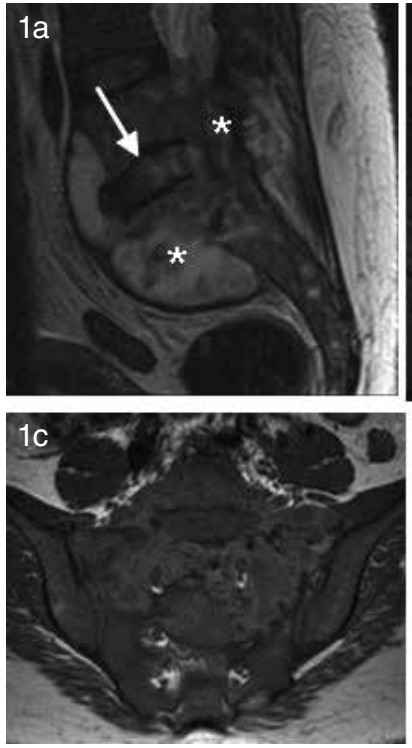
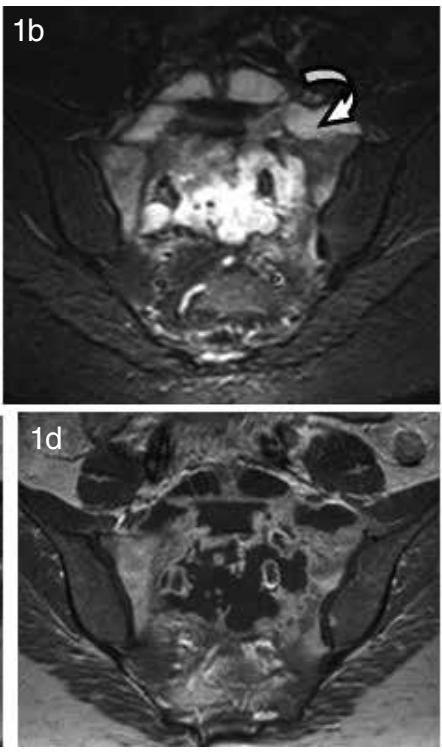
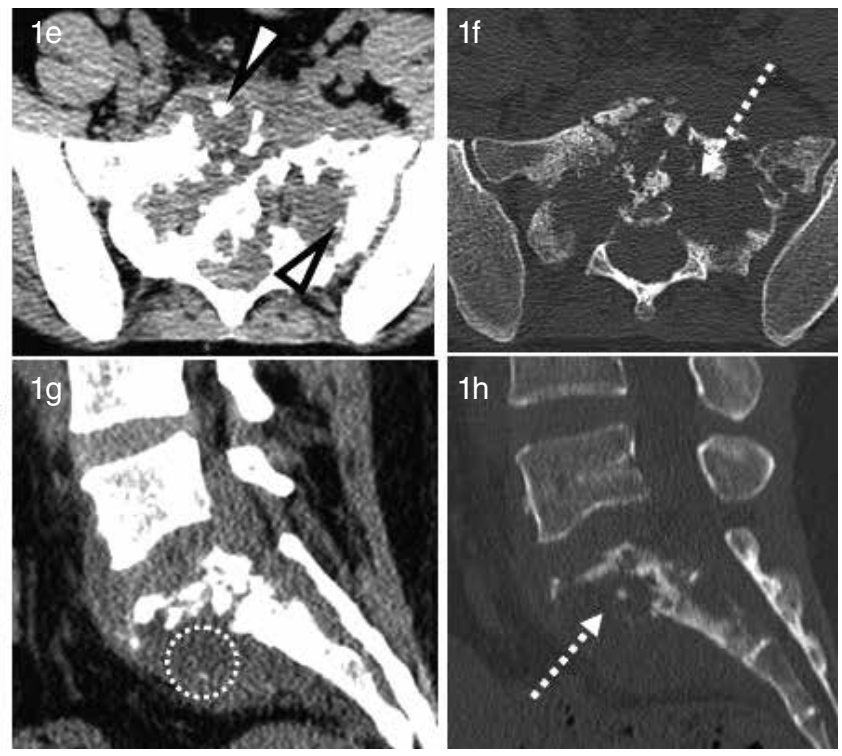

Figura 1. Imágenes en planos sagitales y coronales sobre el sacro. RM en secuencias T2 (a), STIR (b), T1 (c) y T1 con contraste (d); $y$ de TC sin contraste (e-h). Se observa una masa en la unión lumbosacra muy hiperintensa en T2 y STIR e hiper-isointensa en T1. En STIR se observan niveles líquido-líquido (flecha curva en b). Capta el contraste en periferia. En el TC es hipodensa (círculo en g) y tiene pequeñas calcificaciones en su interior (cabezas de flecha en e). Hay signos de destrucción ósea sacra (flechas discontinuas en $f$ y h), afectación del soma L5 y discal L5-S1 (flecha en a), e importante componente de partes blandas que ocupa espacios presacro y epidural anterior (asteriscos en a).

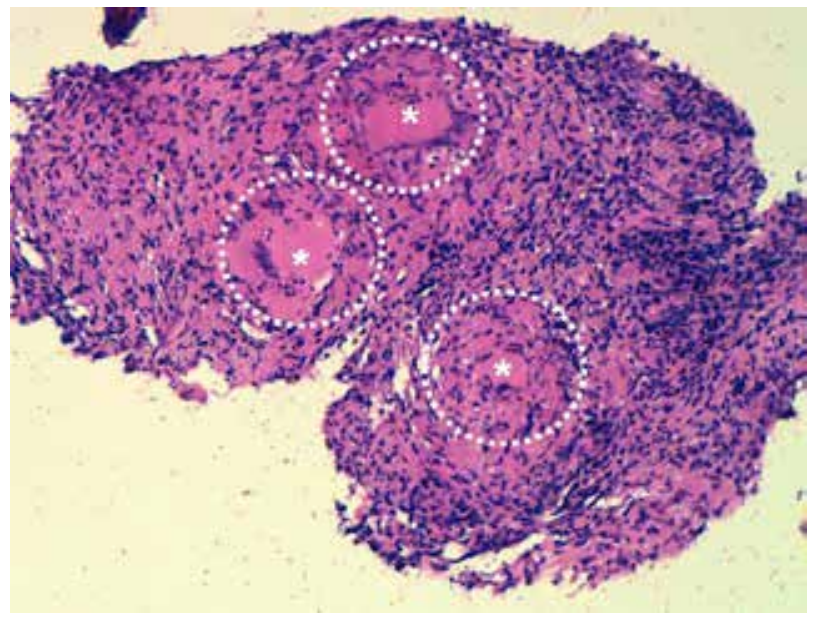

Figura 2. Granulomas de mediano tamaño (círculos) formados por histiocitos epitelioides y células gigantes multinucleadas con áreas microscópicas centrales de necrosis (asteriscos). Se encuentran sobre un fondo de fibrosis e infiltrado inflamatorio crónico de predominio linfocitario.

\section{Caso 2}

Varón de 81 años sin antecedentes de interés. Consultó por cuadro de dolor cervical de meses de evolución.

La TC mostró una voluminosa tumoración en la charnela cráneo-cervical con extensa destrucción ósea y extensión a partes blandas que condicionaba una estenosis secundaria de canal raquídeo. El seno lesional destacaba por su baja densidad y por la presencia de calcificaciones intralesionales (Figura 3).
Los diagnósticos considerados fueron por la vertiente tumoral: el cordoma, el condrosarcoma y las metástasis. Por la vertiente pseudo-tumoral se plantearon: artropatía por depósito de pirofosfato cálcico y proceso inflamatorio-infeccioso atípico de carácter granulomatoso.

Se practicó una biopsia guiada por TC obteniéndose también aislamiento de Mycobacterium Tuberculosis.

Con el diagnóstico de tuberculosis de la unión cráneo-cervical, se inició tratamiento médico. El paciente empeoró clínicamente y falleció pocas semanas después tras una cirugía descompresiva del cordón.

\section{Discusión}

La tuberculosis es causada por el microorganismo bacilo alcohol-resistente $\mathrm{M}$. Tuberculosis. Con el aumento de las inmunodeficiencias y las migraciones poblacionales, se considera una enfermedad de nuevo emergente en el siglo XXI que sigue siendo más prevalente en países en vías de desarrollo ${ }^{(1-6)}$.

En el $80 \%$ de casos de tuberculosis, la afectación es exclusivamente pulmonar. La enfermedad extrapulmonar es infrecuente e incrementa en los pacientes inmunodeprimidos. Por orden de frecuencia, las localizaciones extrapulmonares son: el sistema linfático, la pleura, el tracto genitourinario, el sistema osteoarticular, el sistema nervioso central, el tracto gastrointestinal, el pericardio y por último la enfermedad sistémica de diseminación hematógena (clásicamente llamada afectación miliar). Dentro del sistema osteoarticular; la localización más frecuente es la vertebral, sobretodo dorsal inferior (enfermedad 

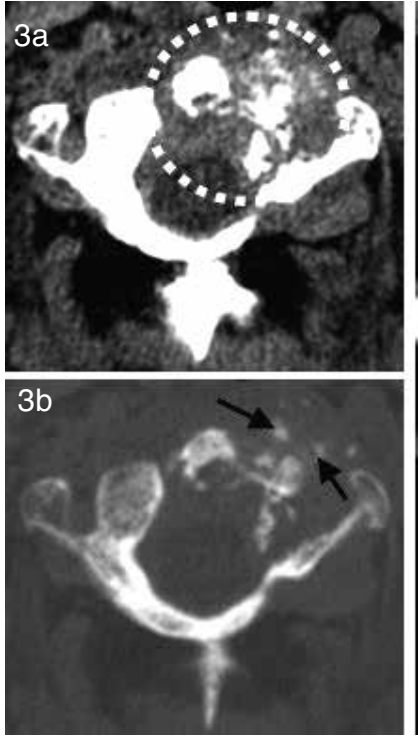
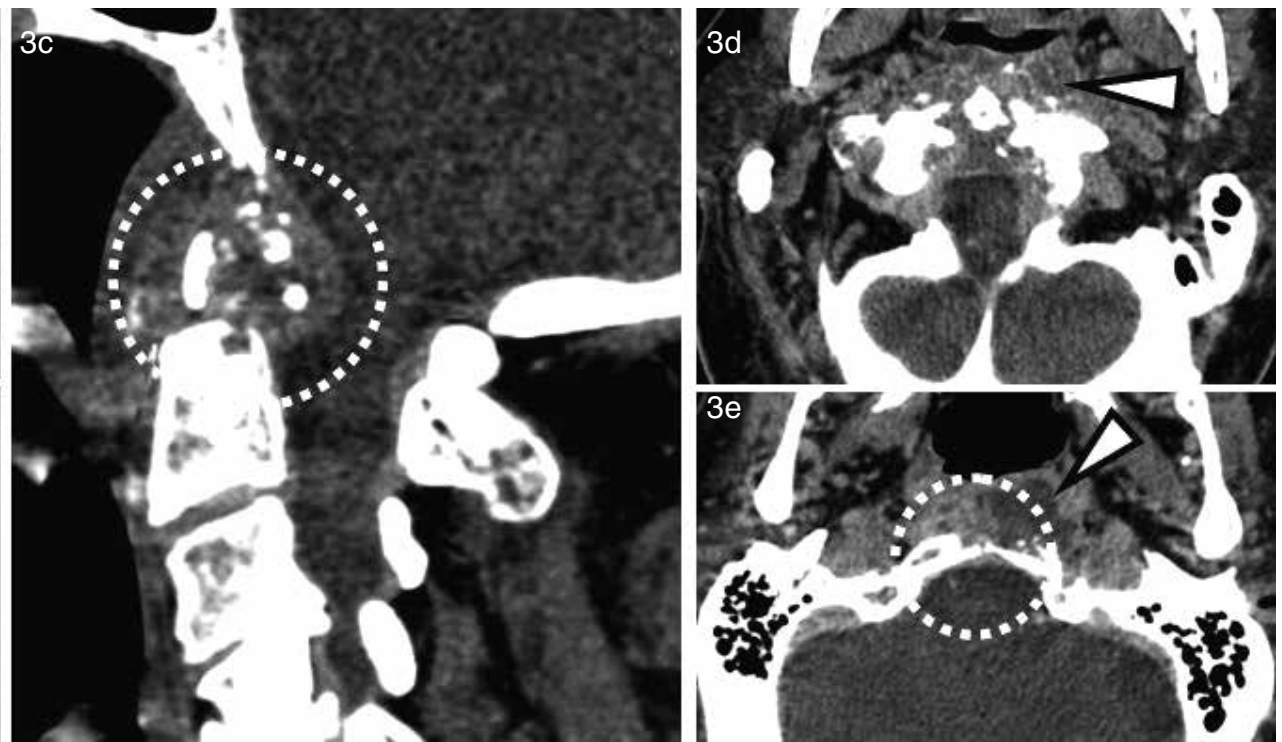

Figura 3. Imágenes en planos sagital (c) y axiales ( $a, b, d$ y e) sobre la unión cráneo-cervical; sin (a-c) y con contraste $(d, e)$. Se observa una lesión destructiva ósea alrededor del odontoides, masa lateral izquierda del atlas y clivus (círculos en c, a y e respectivamente). Es hipodensa (cabeza de flecha en d y e) y tiene pequeñas calcificaciones en su interior (flechas en b y d). Capta el contraste en periferia. Predomina el componente de partes blandas sobre la destrucción ósea.

de Pott o espondilitis tuberculosa) y lumbar. Las dos principales vías de diseminación son la linfática y la hematógena ${ }^{(1)}$.

Los hallazgos radiológicos que podemos encontrar en la enfermedad tuberculosa vertebral son:

- Voluminosas colecciones peri y paravertebrales con calcificaciones, rasgo característico de la enfermedad tuberculosa.

- Afectación de múltiples niveles somáticos a través de su típica extensión subligamentosa, hecho que permite que se afecten varios discos sin grandes fenómenos de afectación ósea en fases avanzadas destrucción y colapso del cuerpo vertebral ${ }^{(1-3,6)}$.

En cuánto al diagnóstico diferencial consideramos: cordoma, condrosarcoma y metástasis.

El cordoma es el principal diagnóstico diferencial en estas dos localizaciones. Es el tumor maligno primario de clivus y sacro más frecuente en adultos. Típicamente se manifiesta como una lesión lítica del hueso con calcificaciones. Suele extenderse a partes blandas y el seno lesional suele ser muy hipodenso en la TC e hiperintenso en T2 de resonancia magnética $(\mathrm{RM})$. Puede también afectar varios niveles vertebrales.

El condrosarcoma se caracteriza por su tendencia a implicar elementos vertebrales posteriores y su aspecto más característico son las calcificaciones de patrón condroide.

Las metástasis óseas en estas localizaciones no son infrecuentes y cabe considerarlas siempre en el diferencial. El antecedente de neoplasia previa sugerirá altamente el diagnóstico.
En el caso lumbo-sacro; también podría considerarse el tumor de células gigantes (TCG) con quiste óseo aneurismático (QOA). El TCG se caracteriza primariamente por su hipointensidad en T2 y su ávida captación del contraste; puede tener componente de QOA secundario que se caracteriza por la presencia de niveles hemáticos.

La región atlo-axoidea es una localización típica de la artropatía por depósito de pirofosfato cálcico. Los fenómenos de condrocalcinosis y las calcificaciones de los ligamentos periodontoideos son las características que radiológicamente pueden orientar a este diagnóstico. Raramente cursa con grandes erosiones óseas y derrame articular; en este caso puede simular cierta agresividad(1-4,6-10).

Los dos casos presentados ilustran localizaciones vertebrales atípicas de la enfermedad tuberculosa. Su rareza y la ausencia de signos de afectación pulmonar o en otras localizaciones relativamente típicas los hizo de difícil diagnóstico.

Así pues, el interés de los casos expuestos radica en la excepcionalidad de estas dos localizaciones y en la importancia de saber reconocer la tuberculosis en cualquier parte del cuerpo. Debemos conocer las peculiaridades que podemos encontrar en cada situación y saber que en algunos casos puede simular malignidad. En un ámbito clínico y epidemiológico adecuado, se debe considerar el diagnóstico de tuberculosis de la columna vertebral ante todo proceso espondilodiscítico con: gran afectación de partes blandas en forma de colecciones con calcificaciones, extensión subligamentaria a múltiples somas vertebrales y proporcional escasa destrucción ósea. 


\section{Bibliografía}

1. Moon KW, Han MH, Chang KH, Im JG, Kim HJ, Sung KJ, Lee HK. CT and MR Imaging of Head and Neck Tuberculosis. RadioGraphics 1997; 17: 391-402.

2. Engin G, Acunas B, Acunas G, Tunaci M. Imaging of Extrapulmonary Tuberculosis. RadioGraphics 2000; 20: 471-488.

3. Yu Y, Wang X, Du B, Yuan W, Ni B, Chen D. Isolated atypical spinal tuberculosis mistaken for neoplasia: case report and literature review. Eur Spine J 2013; 22(Suppl 3): S302-S305.

4. Patankar T, Krishnan A, Patkar D, Kale H, Prasad $\mathrm{S}$, Shah J, Castillo M. Imaging in isolated sacral tuberculosis: a review of 15 cases. Skeletal Radiology 2000; 29: 392-396.

5. Qureshi MA, Afzal W, Khalique AB, Pasha IB, Aebi $M$. Tuberculosis of the craniovertebral junction. Eur Spine J 2013; 22(Suppl 4): S612-S617.
6. Sament R, Bachhal V, Gopinathan NR, Kumar Sen R. Isolated Tuberculosis of Sacrum with Monoparesis: An Atypical Presentation. Asian Spine J 2013; 7(4): 351-354.

7. Shantanu K, Sharma V, Kumar S, Jain S. Tuberculosis of sacrum mimicking as malignancy. BMJ Case Reports 2012; doi: 10.1136/bcr.07.2011.4505.

8. Kumar N, Kumar Gadpayle A, Sundriyal D, Trisal D. Craniovertebral junction tuberculosis. BMJ Case Rep 2013. doi: 10.1136/bcr-2013-202136.

9. Behari S, Nayak SR, Bhargava V, et al. Craniocervical tuberculosis: Protocol of surgical management. Neurosurgery 2003; 52: 72-80.

10. Rodallec $M H$, Feydy $A$, Larousserie $F$, Anract $P$, Campagna R, Babinet A, Zins M, Drapé JL. Diagnostic Imaging of Solitary Tumors of the Spine: What to Do and Say. RadioGraphics 2008; 28: 1019-1041. 